\title{
PREDICT: model for prediction of survival in localized prostate cancer
}

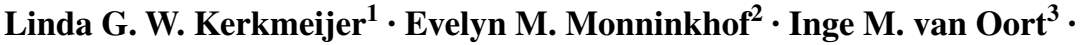 \\ Henk G. van der Poel ${ }^{4}$ Gert de Meerleer ${ }^{5} \cdot$ Marco van Vulpen $^{1}$
}

Received: 16 February 2015 / Accepted: 14 September 2015 / Published online: 29 September 2015

(c) The Author(s) 2015. This article is published with open access at Springerlink.com

\begin{abstract}
Purpose Current models for prediction of prostate cancer-specific survival do not incorporate all present-day interventions. In the present study, a pre-treatment prediction model for patients with localized prostate cancer was developed.

Methods From 1989 to 2008, 3383 patients were treated with I-125 brachytherapy $(n=1694)$, external beam radiotherapy ( $\geq 74 \mathrm{~Gy}, n=336$ ) or radical prostatectomy ( $n=1353$ ). Pre-treatment parameters (clinical T-stage, biopsy grade, PSA and age) were related to the hazard of mortality by multivariate Cox proportional hazard analysis. The PRetreatment Estimation of the risk of Death In Cancer of the prosTate (PREDICT) model was developed. The predictive accuracy of the model was assessed by calibration and discrimination and compared to the Ash risk classification system.

Results Of the 3383 patients analyzed, 2755 patients $(81 \%)$ were alive at the end of follow-up, 149 patients (4\%) died of prostate cancer and 365 patients $(11 \%)$ died

Linda G. W. Kerkmeijer

L.Kerkmeijer@umcutrecht.nl

1 Department of Radiation Oncology, University Medical Center Utrecht, Heidelberglaan 100, 3584 CX Utrecht, The Netherlands

2 Julius Center for Health Sciences and Primary Care, University Medical Center Utrecht, Utrecht, The Netherlands

3 Department of Urology, Radboud University Medical Center, Nijmegen, The Netherlands

4 Department of Urology, Antoni van Leeuwenhoek Hospital, Netherlands Cancer Institute, Amsterdam, The Netherlands

5 Department of Radiation Oncology, Ghent University Hospital, Ghent, Belgium
\end{abstract}

of other causes, and for 114 patients (3\%) cause of death was unknown. Median follow-up time was 7.6 years. After correction for overoptimism, the $c$-statistic of the prediction model for prostate cancer-specific mortality was 0.78 (95\% CI 0.74-0.82), compared to 0.78 (95\% CI 0.750.81 ) for the risk classification system by Ash et al. The PREDICT model showed better calibration than the Ash risk classification system.

Conclusions The PREDICT model showed a good predictive accuracy and reliability. The PREDICT model might be a promising tool for physicians to predict diseasespecific survival prior to any generally accepted intervention in patients with localized prostate cancer.

Keywords Grade Prediction - Prostate cancer PSA · Survival $\cdot$ T-stage

\section{Introduction}

Many models have been developed for risk stratification of patients with localized prostate cancer. Most nomograms incorporate pre-treatment PSA, clinical T-stage and biopsy Gleason score and are developed to predict biochemical failure for patients undergoing radical prostatectomy (RP), based on either pre-treatment clinical parameters [1-3] or post-treatment clinical parameters [4]. Other nomograms were developed for pre-treatment prediction of biochemical recurrence after external beam radiotherapy (EBRT) [5] or brachytherapy [6]. In some studies, prediction was based on patients treated with either RP or radiotherapy [7]. Although prediction of biochemical failure is important, survival outcome after biochemical recurrence is highly heterogeneous. Since disease-specific survival (DSS) is a more representative measure for significant disease 
outcome, currently available biochemical risk nomograms are subject of discussion. Generally accepted present-day interventions for localized prostate cancer include I-125 brachytherapy, RP or high-dose ( $\geq 74$ Gy) EBRT [8-10]. Predictive models that have focused on survival as an outcome measure do not include all present-day standard treatment modalities [11-13]. Other models not included generally accepted current treatment options exclusively [14, 15] and may therefore underestimate survival outcome after treatment in case less effective treatment modalities are included in the model design. In daily practice, due to its general applicability and easy use, risk categories (low, intermediate and high risk) instead of models are frequently used for determining the preferred treatment, for instance the scoring system of Ash and the D'Amico risk classification system, both developed for prediction of biochemical recurrence $[16,17]$. The risk category systems, however, do not allow prediction of individual patient survival outcome accurately [18]. In the present study, we aimed to develop a multivariate simple model for prediction of DSS and overall survival (OS) for localized prostate cancer patients prior to treatment with standard present-day interventions (RP, I-125 brachytherapy or high-dose EBRT). The following pre-treatment parameters were taken into account: clinical T-stage, initial PSA (iPSA), biopsy tumor grade and patient age.

\section{Patients and methods}

\section{Patients and follow-up}

Clinical data were collected prospectively for patients treated in four university hospitals in The Netherlands and Belgium. Only patients treated up till 2008 were included in the present study, since sufficient follow-up was required to determine the 10-year probability of DSS and OS. From 1989 to 2008, 1694 patients were treated at the UMC Utrecht Radiotherapy department with Iodine-125 brachytherapy (145 Gy). Implantations were performed according to the GEC-ESTRO guidelines [16]. In the years 1991 up till 2008, 1353 patients were treated by prostatectomy at the Urology Department of the Radboud University Medical Center and the Netherlands Cancer Institute. The method of RP varied (open or laparoscopic or robot-assisted surgery and nerve sparing or non-nerve sparing). Among these 1353 patients, $335(25 \%)$ have been treated by adjuvant radiotherapy. Between 1998 and 2008, 336 patients were treated at the Ghent University Hospital using an intensitymodulated radiotherapy technique (IMRT) to a dose of 75-78 Gy.

Follow-up was measured until death or the latest known date of being alive (censor date). For the Dutch patients, survival status was retrieved through linkage with the Dutch Cancer Registry at the 31st of December 2012. For the Belgian patients, survival data were derived from the hospitals follow-up program and information from general physicians up till July 2013. Cause of death was retrieved from the medical charts.

\section{Pre-treatment predictors}

Clinical data were derived from the patient's medical records. Predictive clinical parameters were pre-specified based on the literature to prevent overfitting. Pretreatment T-stage, grade (throughout time different Gleason score/tumor grade definitions have been used), initial PSA (iPSA) and age were incorporated in the PREDICT model. The American Joint Committee TNM classifications were used for determination of the T-stage [19]. To compare the predictive accuracy of the prognostic model with standard risk classification systems, patients were also classified by the Ash classification system (low-risk: T1c-T2a, PSA $<10 \mathrm{ng} / \mathrm{mL}$, Gleason score $\leq 6$; intermediate-risk: T2b-T2c, PSA 10-20 ng/mL, Gleason score 7; high-risk: $\geq$ T3a, PSA $>20 \mathrm{ng} / \mathrm{mL}$, Gleason score $\geq 8$ and/or $\geq 2$ intermediate-risk factors) [16].

\section{Statistics}

Kaplan-Meier analysis was used to estimate the actuarial DSS and OS. Baseline clinical characteristics and treatment modality are listed in Table 1 . For 263 patients (7\%), one or more clinical parameters could not be retrieved (missing completely at random). Imputed missing clinical parameters were used by single imputation to prevent bias that would have occurred if only complete cases were used in the analysis [20]. When compared to the complete case
Table 1 Clinical baseline characteristics of localized prostate cancer patients treated by brachytherapy, prostatectomy and external beam radiotherapy

\begin{tabular}{lllll}
\hline & Brachytherapy & Prostatectomy & EBRT & Total \\
\hline No. of patients & 1694 & 1353 & 336 & 3383 \\
Age (years, mean, range) & $66(43-91)$ & $61(42-80)$ & $66(41-80)$ & $64(41-91)$ \\
iPSA (ng/mL, median, range) & $8.9(0.3-100)$ & $8.0(0.1-157)$ & $10.3(0.04-302)$ & $8.7(0.04-302)$ \\
Clinical T-stage (median, range) & $\mathrm{cT} 1(\mathrm{cT} 1-\mathrm{cT} 2 \mathrm{c})$ & $\mathrm{cT} 2 \mathrm{a}(\mathrm{cT} 1-\mathrm{cT} 4)$ & $\mathrm{cT} 2 \mathrm{a}(\mathrm{cT} 1-\mathrm{cT} 4)$ & $\mathrm{cT} 1(\mathrm{cT} 1-\mathrm{cT} 4)$ \\
Biopsy grade (median, range) & $1(1-3)$ & $1(1-3)$ & $1(1-3)$ & $1(1-3)$ \\
\hline
\end{tabular}


analyses (Table 1), measures of central tendency and dispersion did not change significantly after imputation. Survival outcome was not imputed. The potential predictive parameters including $\mathrm{T}$-stage $(\mathrm{T} 1, \mathrm{~T} 2$ and $\geq \mathrm{T} 3 \mathrm{a})$, grade (1, 2 and 3), iPSA (log PSA) and age (age at treatment per 5 years) were related to the hazard of mortality by means of multivariate Cox regression. Cox regression analysis was used for its ability to use censored observations. The values for iPSA and age automatically centered around the mean in the statistical analysis (therefore a negative number of points can be obtained). The ability of the model to discriminate between 10 -year mortality or survival was calculated by means of $c$-statistics (Harrell's concordance index). Since a model's predictive accuracy is generally too optimistic, internal validation was performed by bootstrapping $(n=200)$ to correct for this overfitting [21]. When using bootstrapping techniques, a model's performance better reflects the expected performance in a new population of patients. By means of shrinkage of the $\beta$-coefficients, the model was adjusted for this overoptimism. The final model was transformed into a clinical prediction rule based on the $\beta$-coefficients after shrinkage. The predictive accuracy of the final PREDICT model was evaluated by calibration (reliability) and discrimination. Calibration was assessed by plotting the deciles of the predicted risks of survival (median predicted percentage per decile) compared with the observed risks of survival (10-year DSS derived from actuarial life tables Kaplan-Meier analysis). The prognostic performance of the PREDICT model was compared to that of the risk classification system for prostate cancer by Ash et al. [16].

Descriptive statistics and imputation were performed with the Statistical Package for Social Sciences (SPSS), version 20.0 (Chicago, IL, USA). All other statistical analyses were carried out in the statistical package of $R$ version 2.10 (free software from the GNU project).

\section{Results}

Of the 3383 patients analyzed, 2755 patients $(81 \%)$ were alive at time of the censor date (31st of December 2012), 618 patients $(18 \%)$ died, and for 10 patients follow-up of survival status could not be retrieved. Among the 618 patients who did not survive, 149 patients $(4 \%)$ died of prostate cancer and 365 patients $(11 \%)$ died of nonprostate cancer-related death, and for 114 patients (3\%) cause of death was not known. Median follow-up time was 7.6 years (mean 8.3 years, SD 3.8 years), and 958 (28\%) patients reached 10.0-years follow-up (Table 2). According to the Ash risk classification system, 1198 (35\%) patients were categorized as having low-risk prostate cancer, $1151(31 \%)$ as having intermediate-risk disease and 1034 (34\%) as having high-risk cancer.

Multivariate Cox regression analyses showed a $c$-statistic (before bootstrapping) of the PREDICT model for prediction of DSS of 0.81 (SD 0.03) and 0.68 (SD 0.02) for prediction of OS. In comparison, the corresponding $c$-statistics were 0.78 (SD 0.03) for DSS and 0.61 (SD 0.02) for OS for the risk classification system by Ash et al. The model was corrected for overoptimism (4\% for DSS and $2 \%$ for OS). For the Ash risk scoring system, 0 versus $1 \%$ overoptimism was found. The adjusted $\beta$-coefficients for the PREDICT model for prediction of DSS after shrinkage are shown in Table 3 . The $\beta$-coefficients were rounded (multiplied by 10 ) to obtain a practical prediction rule. The $c$-statistics of the final PREDICT model after bootstrapping was 0.78 (95\% CI 0.74-0.82) for disease-specific survival. In comparison, a $c$-statistics of 0.78 (95\% CI 0.75-0.81)

Table 3 Multivariable predictors (after shrinkage) of disease-specific survival for prostate cancer for the PREDICT model

\begin{tabular}{lll}
\hline Predictor & HR & $95 \%$ CI \\
\hline Age & & \\
$\quad$ Age per 5 years & 1.2 & $1.1-1.4$ \\
Pretreatment T-stage & & \\
$\quad$ cT1 (reference) & & \\
cT2 & 1.7 & $1.2-2.5$ \\
$\geq$ cT3a & 1.8 & $0.9-3.6$ \\
Pretreatment grade & & \\
G1 (reference) & & \\
G2 & 2.9 & $2.0-4.2$ \\
G3 & 3.6 & $2.1-6.1$ \\
Pretreatment PSA & & $2.6-6.4$ \\
Log PSA & 4.1 & \\
\hline
\end{tabular}

Table 2 Follow-up, diseasespecific and overall survival for patients with localized prostate cancer per treatment modality (brachytherapy, prostatectomy and external beam radiotherapy)

\begin{tabular}{lllll}
\hline & Brachytherapy & Prostatectomy & EBRT & Total \\
\hline No. of patients & 1694 & 1353 & 336 & 3383 \\
Follow-up (years, mean, 95 \% CI) & $7.9(7.7-8.0)$ & $9.1(8.8-9.3)$ & $7.1(6.8-7.4)$ & $8.3(8.1-8.4)$ \\
10 year DSS (95 \% CI) & $91 \%(90-92)$ & $96 \%(95-97)$ & $93 \%(90-95)$ & $94 \%(93-95)$ \\
10 year OS (95\% CI) & $72 \%(70-74)$ & $87 \%(85-89)$ & $77 \%(72-81)$ & $79 \%(78-80)$ \\
\hline
\end{tabular}

$D S S$ disease-specific survival, $O S$ overall survival 
was calculated for the Ash risk score. For prediction of overall survival, a $c$-statistic of 0.68 (95\% CI 0.65-0.70) was found for the PREDICT model, compared to 0.61 (95\% CI 0.58-0.63) for the scoring system by Ash et al. Figure 1 shows the calibration of the predicted versus the observed risk of disease-specific survival (compared in risk strata) for the PREDICT model versus the Ash risk scoring system.

The total risk score of the PREDICT model for predicting disease-specific survival is calculated by summing up the number of points obtained for each clinical factor (age

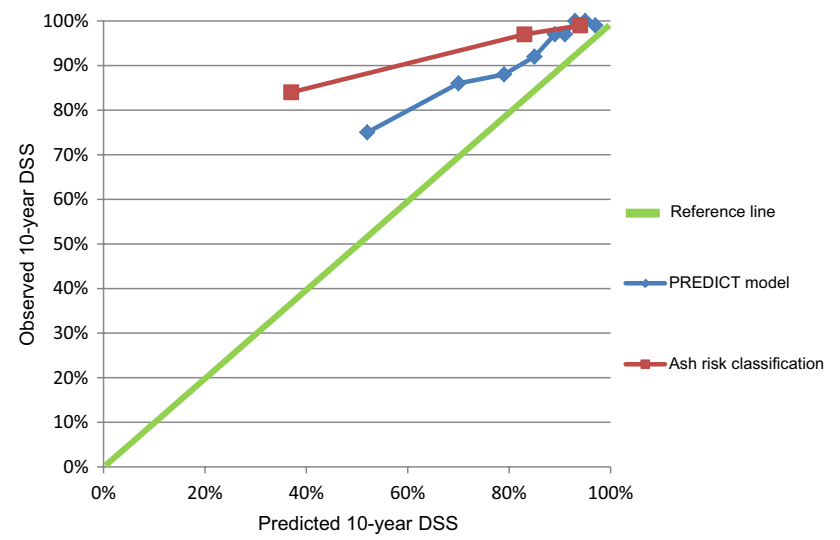

Fig. 1 Calibration plots for prediction of disease-specific survival for the PREDICT model versus the Ash risk classification system. The predicted probability of survival based on the PREDICT model was compared to the observed survival. On the $x$-axis the predicted probability and on the $y$-axis the observed (actuarial life table) probability of 10-year disease-specific survival is shown. A slope of ' 1 ' (reference line) is associated with optimal calibration
+ T-stage + grade + iPSA $=$ total score; Table 4$).$ In this table, also the corresponding predicted 10-year diseasespecific survival rate can be found for different calculated total risk score categories.

\section{Discussion}

After diagnosis of prostate cancer, patients are typically classified into risk categories bases on T-stage, Gleason score and pre-treatment PSA level, most frequently by using the Ash or D'Amico risk classification system [16, 17].

The PREDICT model was developed as an easy tool for use in clinical practice. The goal was to achieve a more reliable prediction of disease-specific survival outcome for individual patients than the existing three category risk classification systems. Both the PREDICT model and the Ash system have a good accuracy for pre-treatment prediction of DSS in non-metastasized prostate cancer ( $c$-statistic 0.78). The PREDICT model, however, had a better calibration performance (reliability) than the Ash risk classification system. In other words, the predicted 10-year DSS is closer to the observed 10-year Kaplan-Meier derived DSS for the PREDICT model than for the Ash risk classification.

Since the model was developed on patients treated by well-established present-day types of treatment only (brachytherapy, high-dose EBRT, RP), this model will be applicable to the majority of prostate cancer patients. Accurate prediction of treatment outcome is essential for patient counseling. The PREDICT model was compared to the Ash risk classification, since this risk classification is frequently

Table 4 Pre-treatment tool to predict the 10-year disease-specific survival for patients with localized prostate cancer (PREDICT model)

\begin{tabular}{|c|c|c|c|c|c|c|c|c|c|c|c|c|c|c|c|}
\hline \multicolumn{16}{|l|}{ Age $(\text { years })^{\mathrm{a}}$} \\
\hline Value & 44 & 49 & 54 & 59 & 64 & 69 & 74 & & 79 & 84 & & 89 & & & \\
\hline Score & -8 & -6 & -4 & -2 & 0 & 2 & 4 & & 6 & 8 & & 10 & & & \\
\hline \multicolumn{16}{|l|}{ T-stage } \\
\hline Value & & & & & $\mathrm{cT} 1$ & & & & $\geq \mathrm{cT} 2$ & & & & & & \\
\hline Score & & & & & 0 & & & & 6 & & & & & & \\
\hline \multicolumn{16}{|l|}{ Grade } \\
\hline Value & & & & & G1 & & & & & & & & $\mathrm{G} 2$ & G3 & \\
\hline Score & & & & & 0 & & & & & & & & 11 & 13 & \\
\hline \multicolumn{16}{|l|}{ iPSA $(n g / m L)^{a}$} \\
\hline Value & & 2 & 3 & 6 & 9 & 13 & 16 & 20 & 25 & 32 & 38 & & 50 & 79 & \\
\hline Score & & -6 & -4 & -2 & 0 & 2 & 4 & 5 & 6 & 8 & 9 & & 11 & 13 & \\
\hline \multicolumn{16}{|l|}{ Total sum score } \\
\hline Total sum score & $<3$ & & $3-7$ & & $8-11$ & & $12-16$ & & $>16$ & & & & & & \\
\hline 10-year DSS (\%) & $\geq 93$ & & $88-92$ & & $82-87$ & & $72-81$ & & $\leq 71$ & & & & & & \\
\hline
\end{tabular}

a Choose the closest value when the patient's value lies between the given values 
used in clinical practice to decide on the treatment modality of preference. Overall survival could not be accurately predicted by the PREDICT model nor by the Ash risk classification. This can be explained by the fact that prostate cancer mortality is only a small proportion of overall mortality, and only prostate cancer-related factors (not comorbidity parameters, except for patient age) were incorporated in the present model design. Walz et al. [22] did develop a model specifically for predicting 10-year general life expectancy with a high predictive accuracy $(84 \%)$ including age and Charlson comorbidity score. Other models have been used to predict life expectancy for non-prostate cancer-related causes [23].

As we expected, our predictive model allows for better individual prediction of DSS than the Ash risk score system. Risk classification relies on extrapolation of several risk factors in a heterogeneous patient group [18]. The strengths of the PREDICT model are the large patient numbers included. The median follow-up is 7.6 years (mean 8.3 years). Furthermore, the whole spectrum of current interventions and only patients treated by well-established interventions (with assumed comparable cure rates) for prostate cancer were incorporated. The low-, intermediate- and high-risk patient groups according to the Ash risk score system were equally represented in the database. A possible limitation of the present study is that for $3 \%$ of patients cause of death could not be retrieved (equally divided among RP and brachytherapy patients, one missing cause of death among EBRT patients). Furthermore, patients were treated in different centers, with possible differences in pre-treatment imaging, number and system of taking biopsies and pathology evaluation (interobserver variability and Gleason score and tumor grade definition throughout time). Huang et al. [24] proposed integration of percentage of positive cores in risk stratification models. However, the percentage of positive cores was not available for a large number of patients in our database and could therefore not be used. The effect of treatment modality (brachytherapy, RP or high-dose radiotherapy) on DSS is not known, since no randomized controlled trials are available up to date. The evidence available is conflicting and was based on retrospective data $[25,26]$. In the present study, we have assumed that DSS is comparable in all three treatment modalities and only dependent on risk factors. T-stage was the weakest predictor in the PREDICT model. T-stage and also tumor grading are known to be subject to understanding by random (and extended) biopsies and digital rectal examination/transrectal ultrasound when compared to post-prostatectomy pathology results [27, 28]. Adding MRI-based T-staging may increase the predictive accuracy of a predictive model. Prediction of biochemical recurrence after EBRT indeed improved by MRI imaging in addition to the Kattan model, when compared to the Kattan model alone [29].
Originally, pre-treatment risk category systems and models were developed to predict biochemical disease control. The Kattan, Stephenson and CAPRA models are the ones mostly used and have been extensively externally validated $[1-3,30]$. A significant proportion of patients with biochemical recurrence, however, does not die from prostate cancer; therefore, endpoints for unambiguous disease outcome such as survival or risk of metastatic disease are more relevant, but require longer follow-up time. Although many predictive models for prostate cancer exist, a minority of models is developed to predict survival and there is a lack of models for survival prediction incorporating all generally accepted present-day interventions [11-15]. Two CAPRA (Cancer of the Prostate Risk Assessment) scorebased models have been developed. The model design, however, did not exclusively include patients treated by current interventions [14, 15]. Particularly, EBRT dose and technique were heterogeneous in both studies. Radiation therapy patients were included from 1987 on, and a lower dose to the prostate has been associated with an inferior tumor control and a higher risk of metastatic spread [10]. Indeed, the model showed a lower discriminative capacity for prediction of survival in patients treated by radiation compared to the prostatectomy group. In the study of Cooperberg et al. [15] also, patients managed by watchful waiting, active surveillance, primary hormonal therapy and cryotherapy were included, therefore biasing survival outcome to a lower survival rate when using the model for patients treated by more effective modalities. For the Kutikov et al. [14] model, discriminative capability of the model was not described, which makes it hard to evaluate the internal validity of the model. Both CAPRAbased models were not externally validated for prediction of prostate cancer-specific mortality. When developing a model, several important considerations should be taken into account [18]. External validation is the golden standard for evaluating reproducibility and accuracy. The PREDICT model was based on large patient numbers with an intermediate follow-up time, has a good discrimination and calibration, but has to be validated in an external dataset before its clinical application. For (internal) validation purposes [21], we did perform bootstrapping in anticipation of the external validation study, which will be performed on a different dataset.

\section{Conclusion}

Available pre-treatment models developed for prediction of survival after treatment for prostate cancer either not include all present-day interventions or include suboptimal treatment options or active surveillance. After external validation, the PREDICT model might be a promising tool for 
physicians to predict survival preceding any current generally accepted intervention for non-metastasized prostate cancer.

Acknowledgments L.G.W. Kerkmeijer and E.M. Monninkhof had full access to all of the data in the study and take responsibility for the integrity of the data and the accuracy of the data analysis. The authors thank the registration teams of the Comprehensive Cancer Centre Netherlands and Comprehensive Cancer Centre South for the collection of data for the Netherlands Cancer Registry and the scientific staff of the Comprehensive Cancer Centre Netherlands. The authors thank Linda M. Peelen, M.Sc., Ph.D., of the Julius Center for Health Sciences and Primary Care, University Medical Center Utrecht for her statistical advise on the development of a prediction model.

Authors' contribution L.G.W. Kerkmeijer: Project development, Data collection, Data analysis and interpretation, Manuscript writing; E.M. Monninkhof: Project development, Data analysis and interpretation, Manuscript writing; I.M. van Oort: Data collection and interpretation, Manuscript editing; H.G. van der Poel: Data collection and interpretation, Manuscript editing; G. de Meerleer: Data collection and interpretation, Manuscript editing; M. van Vulpen: Project development, Data analysis and interpretation, Manuscript writing.

\section{Compliance with ethical standards}

Conflict of interest The authors declare that they have no conflicts of interest.

Ethical standard The manuscript does not include clinical studies or identifiable patient data.

Open Access This article is distributed under the terms of the Creative Commons Attribution 4.0 International License (http://creativecommons.org/licenses/by/4.0/), which permits unrestricted use, distribution, and reproduction in any medium, provided you give appropriate credit to the original author(s) and the source, provide a link to the Creative Commons license, and indicate if changes were made.

\section{References}

1. Kattan MW, Eastham JA, Stapleton AM et al (1998) A preoperative nomogram for disease recurrence following radical prostatectomy for prostate cancer. J Natl Cancer Inst 90:766-771

2. Cooperberg MR, Pasta DJ, Elkin EP et al (2005) The university of California, San Francisco cancer of the prostate risk assessment score: a straightforward and reliable preoperative predictor of disease recurrence after radical prostatectomy. J Urol 173:1938-1942

3. Stephenson AJ, Scardino PT, Eastham JA et al (2006) Preoperative nomogram predicting the 10 -year probability of prostate cancer recurrence after radical prostatectomy. J Natl Cancer Inst 98:715-717

4. Kattan MW, Wheeler TM, Scardino PT (1999) Postoperative nomogram for disease recurrence after radical prostatectomy for prostate cancer. J Clin Oncol 17:1499-1507

5. Zelefsky MJ, Kattan MW, Fearn P et al (2007) Pretreatment nomogram predicting ten-year biochemical outcome of threedimensional conformal radiotherapy and intensity-modulated radiotherapy for prostate cancer. Urology 70:283-287

6. Kattan MW, Potters L, Blasko JC et al (2001) Pretreatment nomogram for predicting freedom from recurrence after permanent prostate brachytherapy in prostate cancer. Urology 58:393-399
7. D'Amico AV, Whittington R, Malkowicz SB et al (2002) Biochemical outcome after radical prostatectomy or external beam radiation therapy for patients with clinically localized prostate carcinoma in the prostate specific antigen era. Cancer 95:281-286

8. Heidenreich A, Bellmunt J, Bolla M et al (2011) EAU guidelines on prostate cancer. Part 1: screening, diagnosis, and treatment of clinically localised disease. Eur Urol 59:61-71 (updated: March 2013, www.uroweb.org)

9. Al-Mamgani A, van Putten WL, Heemsbergen WD et al (2008) Update of Dutch multicenter dose-escalation trial of radiotherapy for localized prostate cancer. Int J Radiat Oncol Biol Phys 72:980-988

10. Zelefsky MJ, Pei X, Chou JF et al (2011) Dose escalation for prostate cancer radiotherapy: predictors of long-term biochemical tumor control and distant metastases-free survival outcomes. Eur Urol 60:1133-1139

11. Eggener SE, Scardino PT, Walsh PC et al (2011) Predicting 15 -year prostate cancer specific mortality after radical prostatectomy. J Urol 185:869-875

12. Stephenson AJ, Kattan MW, Eastham JA et al (2009) Prostate cancer-specific mortality after radical prostatectomy for patients treated in the prostate-specific antigen era. J Clin Oncol 27:4300-4305

13. Teeter AE, Presti JC, Aronson WJ et al (2013) Do nomograms designed to predict biochemical recurrence (BCR) do a better job of predicting more clinically relevant prostate cancer outcomes than BCR? A report from the SEARCH database group. Urology 82:53-59

14. Kutikov A, Cooperberg MR, Paciorek AT et al (2012) Evaluating prostate cancer mortality and competing risks of death in patients with localized prostate cancer using a comprehensive nomogram. Prostate Cancer Prostatic Dis 15:374-379

15. Cooperberg MR, Broering JM, Carroll PR (2009) Risk assessment for prostate cancer metastasis and mortality at the time of diagnosis. J Natl Cancer Inst 101:878-887

16. Ash D, Flynn A, Battermann J et al (2000) ESTRO/EAU/EORTC recommendations on permanent seed implantation for localized prostate cancer. Radiother Oncol 57:315-321

17. D'Amico AV, Whittington R, Malkowicz SB et al (1998) Biochemical outcome after radical prostatectomy, external beam radiation therapy, or interstitial radiation therapy for clinical localized prostate cancer. JAMA 280:969-974

18. Steyerberg EW, Moons KGM, van der Windt DA et al (2013) Prognosis research strategy (PROGRESS) 3: prognostic model research. PLoS Med 10:e1001381

19. Schroder FH, Hermanek P, Denis L et al (1992) The TNM classification of prostate cancer. Prostate Suppl 4:129-138

20. Donders AR, van der Heijden GJ, Stijnen T et al (2006) Review: a gentle introduction to imputation of missing values. J Clin Epidemiol 59:1087-1091

21. Hendriksen JM, Geersing GJ, Moons KG et al (2013) Diagnostic and prognostic prediction models. J Thromb Haemost 11:129-141

22. Walz J, Gallina A, Saad F et al (2007) A nomogram predicting 10-year life expectancy in candidates for radical prostatectomy or radiotherapy for prostate cancer. J Clin Oncol 25:3576-3581

23. Sammon JD, Abdollah F, D'Amico A et al (2015) Predicting life expectancy in men diagnosed with prostate cancer. Eur Urol. doi:10.1016/j.eururo.2015.03.020

24. Huang J, Vicini FA, Williams SG et al (2012) Percentage of positive biopsy cores: a better risk stratification model for prostate cancer? Int J Radiat Oncol Biol Phys 83:1141-1148

25. Sooriakumaran P, Nyberg T, Akre O et al (2014) Comparative effectiveness of radical prostatectomy and radiotherapy in prostate cancer: observational study of mortality outcomes. BMJ 348:g1502 
26. Xiong T, Turner RM, Wei Y et al (2014) Comparative efficacy and safety of treatments for localised prostate cancer: an application of network meta-analysis. BMJ Open 4:e004285

27. Cooke EW, Shrieve DC, Tward JD (2012) Clinical versus pathologic staging for prostate adenocarcinoma. Am J Clin Oncol 35:364-368

28. King CR, McNeal JE, Gill H et al (2004) Extended prostate biopsy scheme improves reliability of Gleason grading: implications for radiotherapy patients. Int J Radiat Oncol Biol Phys 59:386-391
29. Westphalen AC, Koff WJ, Coakley FV et al (2011) Prostate cancer: prediction of biochemical failure after external-beam radiation therapy-Kattan nomogram and endorectal MRI imaging estimation of volume. Radiology 261:477-486

30. Tamblyn DJ, Chopra S, Yu C et al (2011) Comparative analysis of three risk assessment tools in Australian patients with prostate cancer. BJU Int 108:51-57 